\title{
Supporting Information for: Single-Protein Collapse Determines Phase Equilibria of a Biological Condensate
}

\author{
Han-Yi Chou and Aleksei Aksimentiev* \\ Department of Physics, University of Illinois at Urbana-Champaign \\ 1110 W. Green St., Urbana IL, 61801 USA \\ *E-mail: aksiment@illinois.edu
}


a

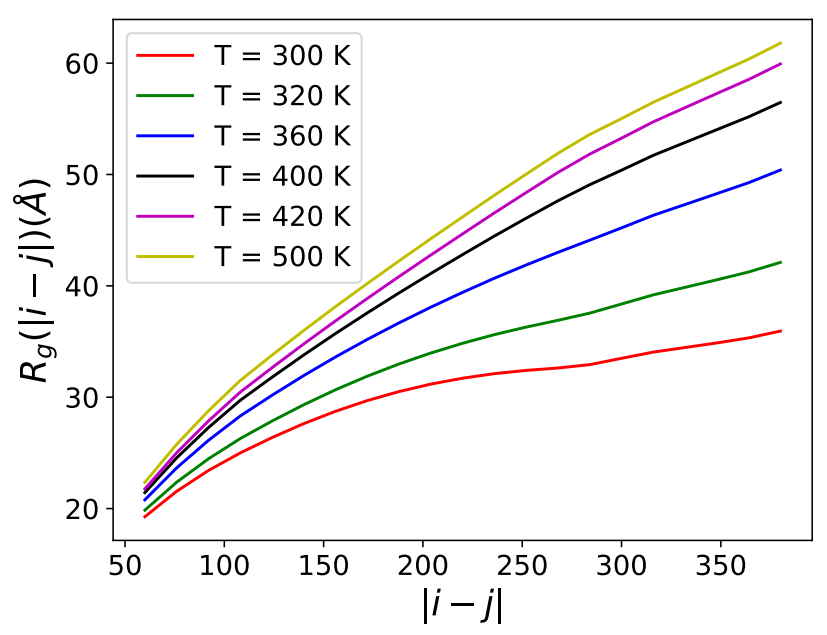

b

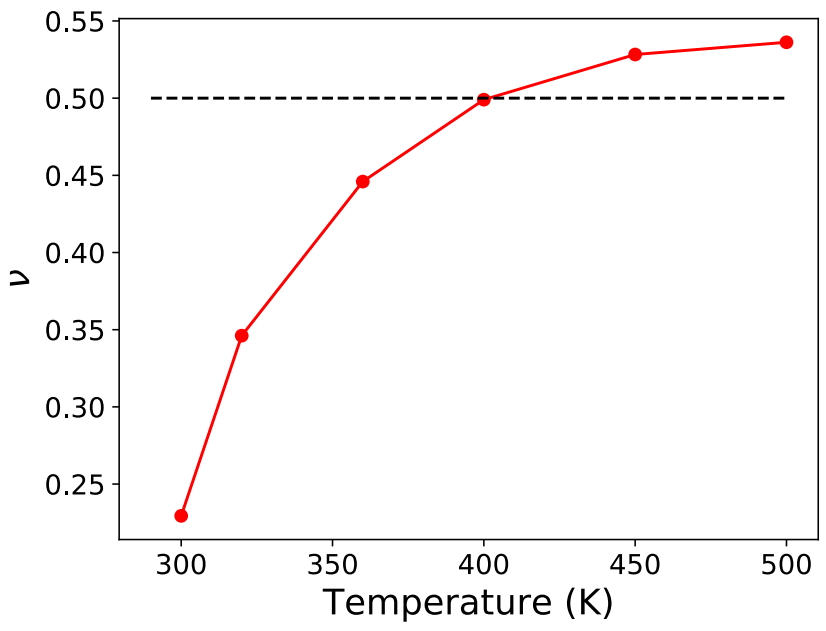

Figure S1: MD simulation of FUS $\theta$ temperature. (a) Simulated radii of gyration of FUS protein fragments as a function of the fragment length, $|i-j|$, where $i$ and $j$ indicate the indices of the fragment's terminal beads. Each $R_{g}(|i-j|)$ value was obtained by averaging over all $(i, j)$ pairs of the same index difference. (b) The scaling exponent $v$ that best describes the asymptotic relation $\left\langle R_{g}(i-j)\right\rangle \sim|i-j|^{v}$ when the difference of the bead indices $|i-j| \rightarrow \infty$. The horizontal dashed line indicates the $v=0.5$ value that corresponds to the $\theta$ temperature. 


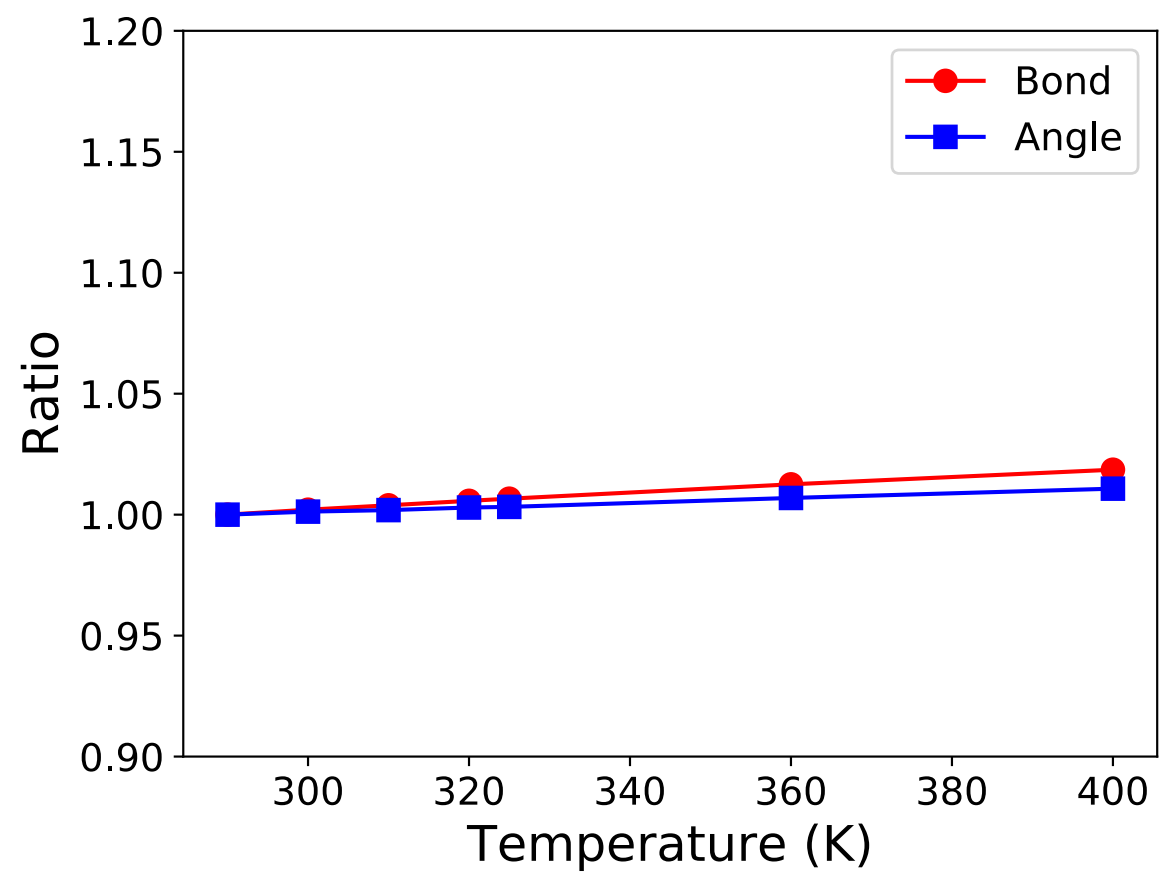

Figure S2: The ratio of the averaged bond length, $l_{0}$ and averaged the bond angle, $\psi$ at different temperatures to the values at $290 \mathrm{~K}$. Each data point was extracted from the last $3 \mu$ s fragment of a $5 \mu$ s MD trajectory of a single FUS protein. 


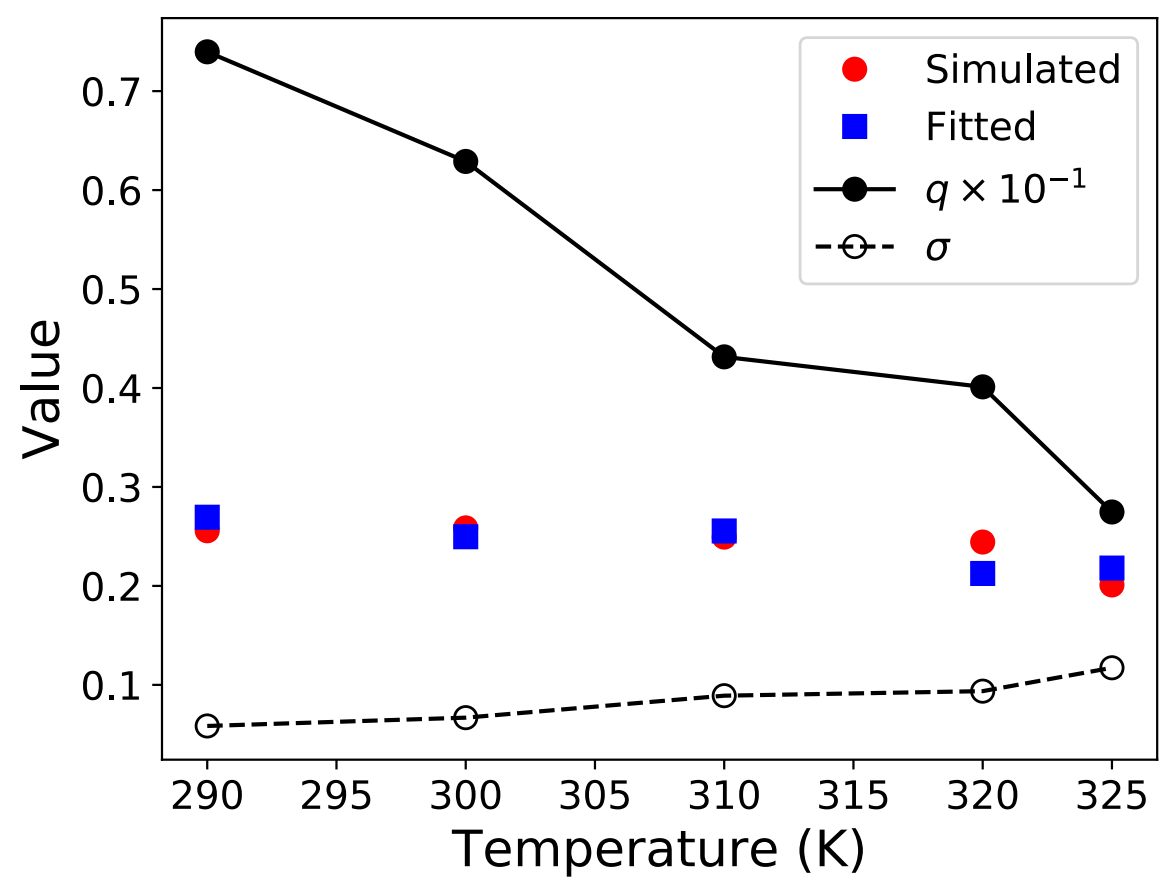

Figure S3: Determination of virial coefficient parameters. Open and solid symbols show the values of $\sigma$ and $q$, respectively, obtained directly from MD simulations of individual FUS proteins at specified temperature. Red circles plot the right-hand side of Eq. [5] evaluated using the $\sigma$ and $q$ obtained form the MD simulations. Blue squares show the values of the left-hand side of Eq. (5) evaluated using the best fit values for $B$ and $K$. The residue of the fitting, i.e., the difference between the right-hand side and the left-hand side of Eq. (5), is 0.04, which corresponds to a relative error of $\sim 7 \%$. 


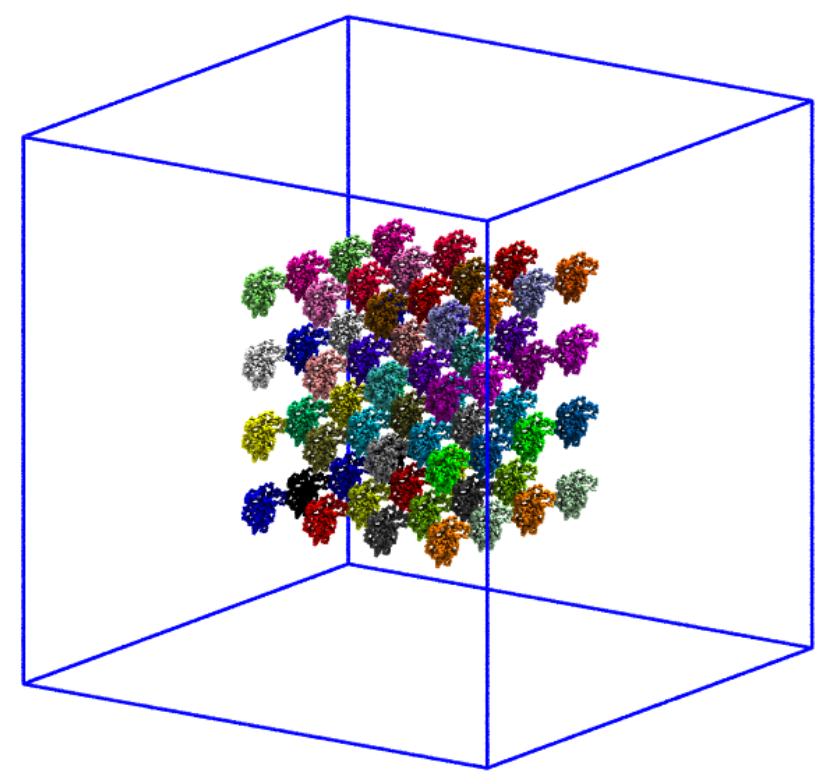

Movie 1: Animation illustrating a CG MD simulation of 64 WT FUS proteins at $292 \mathrm{~K}$, forming a liquid-like droplet after $\sim 1 \mu \mathrm{s}$. 


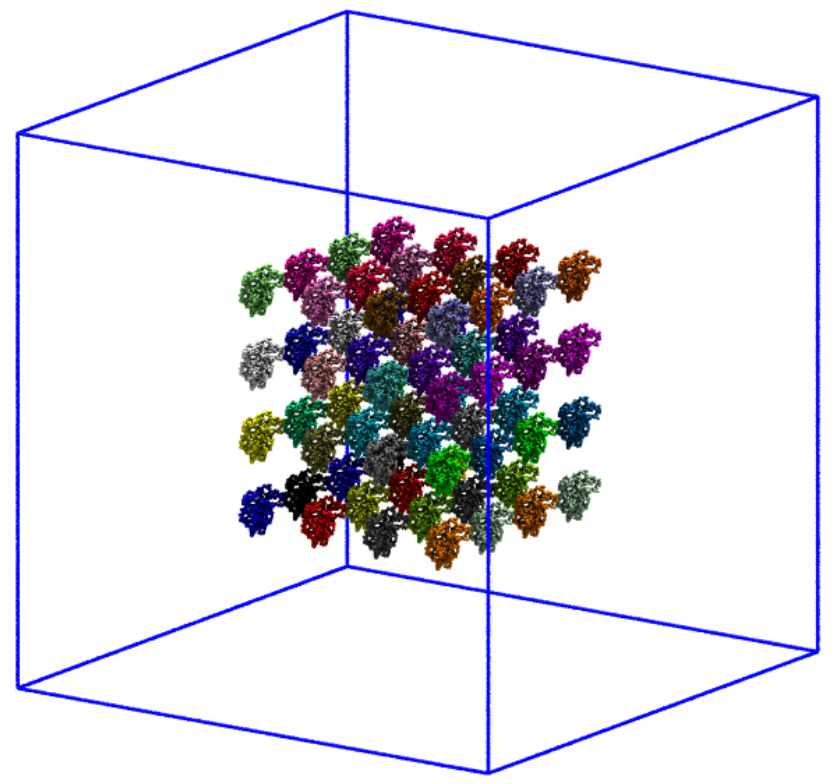

Movie 2: Animation illustrating a CG MD simulation of $64(\mathrm{R} \rightarrow \mathrm{K})$ FUS mutants at $292 \mathrm{~K}$, remaining disperse state after $\sim 1 \mu \mathrm{s}$. 
$325 \mathrm{~K}$
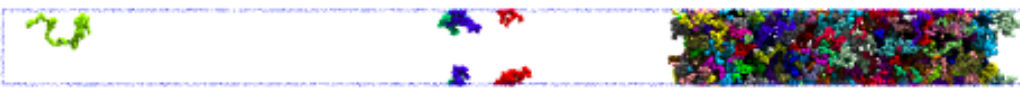

3

$340 \mathrm{~K}$
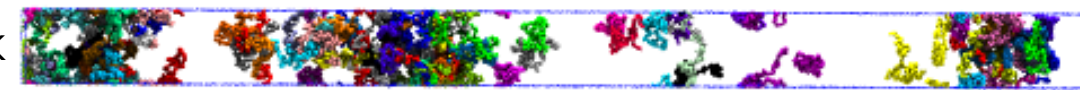

Movie 3: Animation illustrating three CG MD simulations of a slab FUS system performed at different temperatures. Each system contains 125 FUS proteins. 